\title{
Growth Monitoring in Girls Attending a Tertiary Paediatric ENT Service with Middle Ear Disease
}

M Bremner ${ }^{1}$, A Carbarns ${ }^{1}$, I Essa ${ }^{1}$, R Bradley ${ }^{1}$, SC Wong ${ }^{2}$, A Mason ${ }^{2}$, H Kubba ${ }^{3}$

${ }^{1}$ School of Medicine, University of Glasgow, Glasgow, UK; ${ }^{2}$ Developmental Endocrinology Research Group, Royal Hospital for Children, Glasgow, UK; ${ }^{3}$ Department of Paediatric Otolaryngology, Royal Hospital for Children, Glasgow, UK

\section{Background}

Recurrent middle ear disease is very common in childhood although, in most cases, children grow out of the condition as their immune system matures.

In some cases, however, it may lead to poor growth or may suggest an underlying diagnosis associated with short stature. Diagnosis of conditions, such as Turner's syndrome is often delayed and such children may initially present with ENT complaints.

It is important to identify these children as early as possible so that they can be given the appropriate support and treatment.

\section{Aims}

The aim of the study was to describe stature in a cohort of girls attending a Paediatric ENT service with middle ear disease.

\section{Methods}

Height and weight was measured in all girls, including those with comorbidities, attending ENT clinics over an 8-week period $(n=83)$ and converted to SDS using UK 1990 standards.

A mid-parental height (MPH) was calculated from reported parental heights.

Short stature was classified as those girls who had a Height SDS <-2. A deviation from MPH was classified as those who had a difference between MPH and Height SDS >1.5.

The final ENT diagnosis was catergorised as: otitis media with effusion; recurrent acute otitis media; otitis media with perforation; or cholesteatoma.

\section{Results}

For the whole group:

- Median age was $5.89 y r s(0.8,14.5)$

- Median height SDS was $0.09(-4.1,2.9)$

- Median BMI SDS was $0.4(-2.5,4.1)$

\section{Table of Results}

\begin{tabular}{|l|c|c|c|c|}
\hline & $\begin{array}{c}\text { Otitis media } \\
\text { with effusion }\end{array}$ & $\begin{array}{c}\text { Recurrent acute } \\
\text { otitis media }\end{array}$ & $\begin{array}{c}\text { Otitis media } \\
\text { with perforation }\end{array}$ & Cholesteatoma \\
\hline Number of girls & 52 & 23 & 7 & 1 \\
\hline $\begin{array}{l}\text { Median decimal } \\
\text { age(range) }\end{array}$ & $6.0(0.8,14.5)$ & $4.1(1.5,9.8)$ & $9.3(4.7,11.6)$ & 3.7 \\
\hline $\begin{array}{l}\text { Median height } \\
\text { SDS (range) }\end{array}$ & $0.2(-4.1,2.9)$ & $-0.3(-1.3,1.4)$ & $0.3(-1.4,0.7)$ & 0.4 \\
\hline $\begin{array}{l}\text { Median BMI } \\
\text { SDS (range) }\end{array}$ & $0.5(-1.5,4.2)$ & $0.2(-2.9,2.7)$ & $0.5(-1.0,2.2)$ & 2.2 \\
\hline $\begin{array}{l}\text { Median MPH } \\
\text { SDS (range) }\end{array}$ & $0.5(-1.92 .3)$ & $0.11(-1.6,1.8)$ & $-0.2(-1.9,0.9)$ & 0.5 \\
\hline $\begin{array}{l}\text { Median height } \\
\text { SDS - MPH } \\
\text { SDS (range) }\end{array}$ & $-0.04(-3.9,2.9)$ & $-0.6(-2.1,2.6)$ & $-0.2(-1.3,2.4)$ & -0.1 \\
\hline
\end{tabular}

\section{Graph 1: Height SDS}

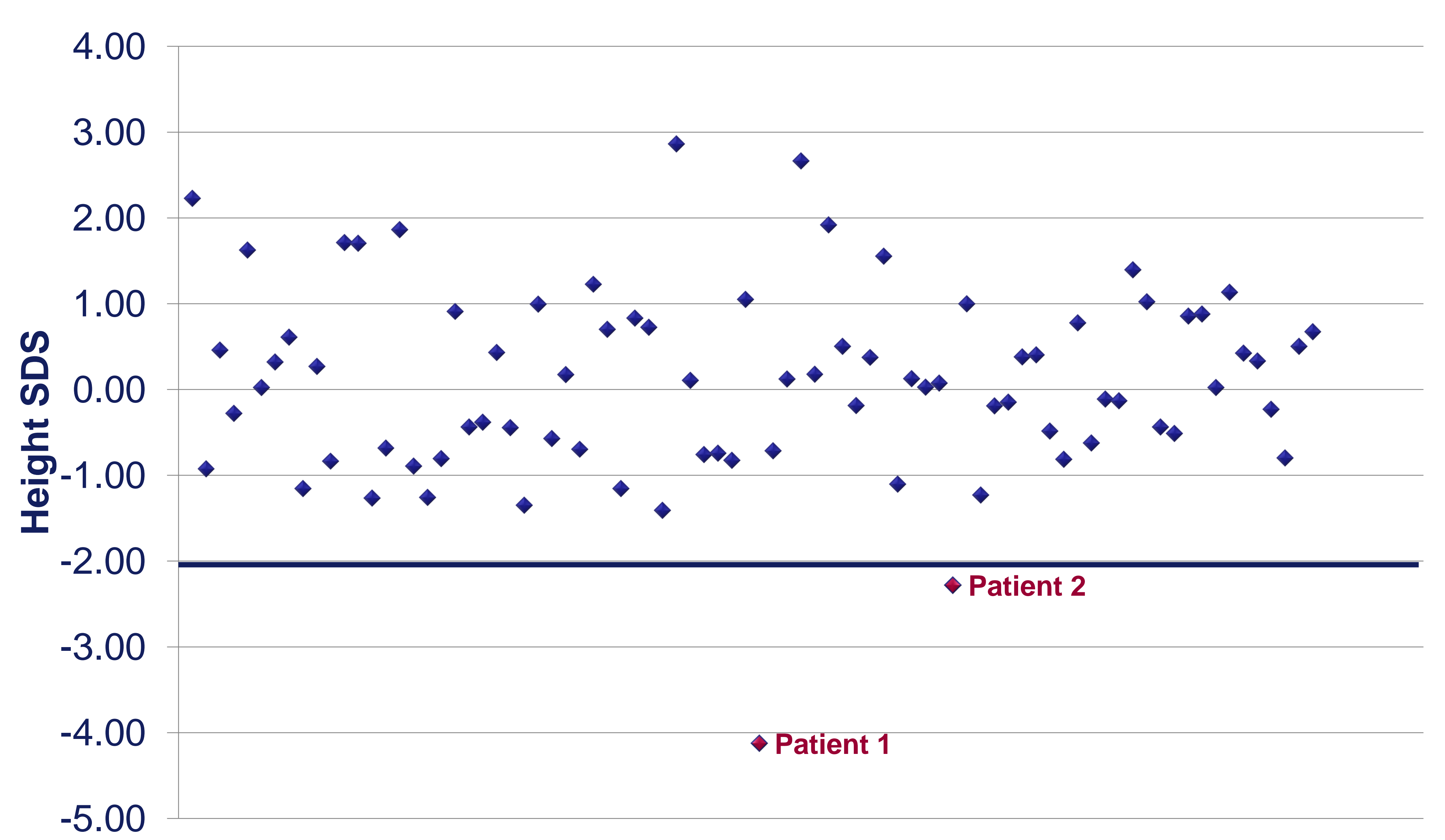

2/83 girls (2.4\%) had height <-2.0SD and >1.5SD lower than MPH SDS Patient 1: Trisomy 21 (14.5yrs, heightSD -4.1, heightSD - MPHSD -3.9).

Patient 2: Recurrent UTI, under general paediatric follow-up and results of investigations pending (10.2yrs, heightSD -2.3, heightSD MPHSD -1.90).

\section{Graph 2: Height SDS - MPH SDS}

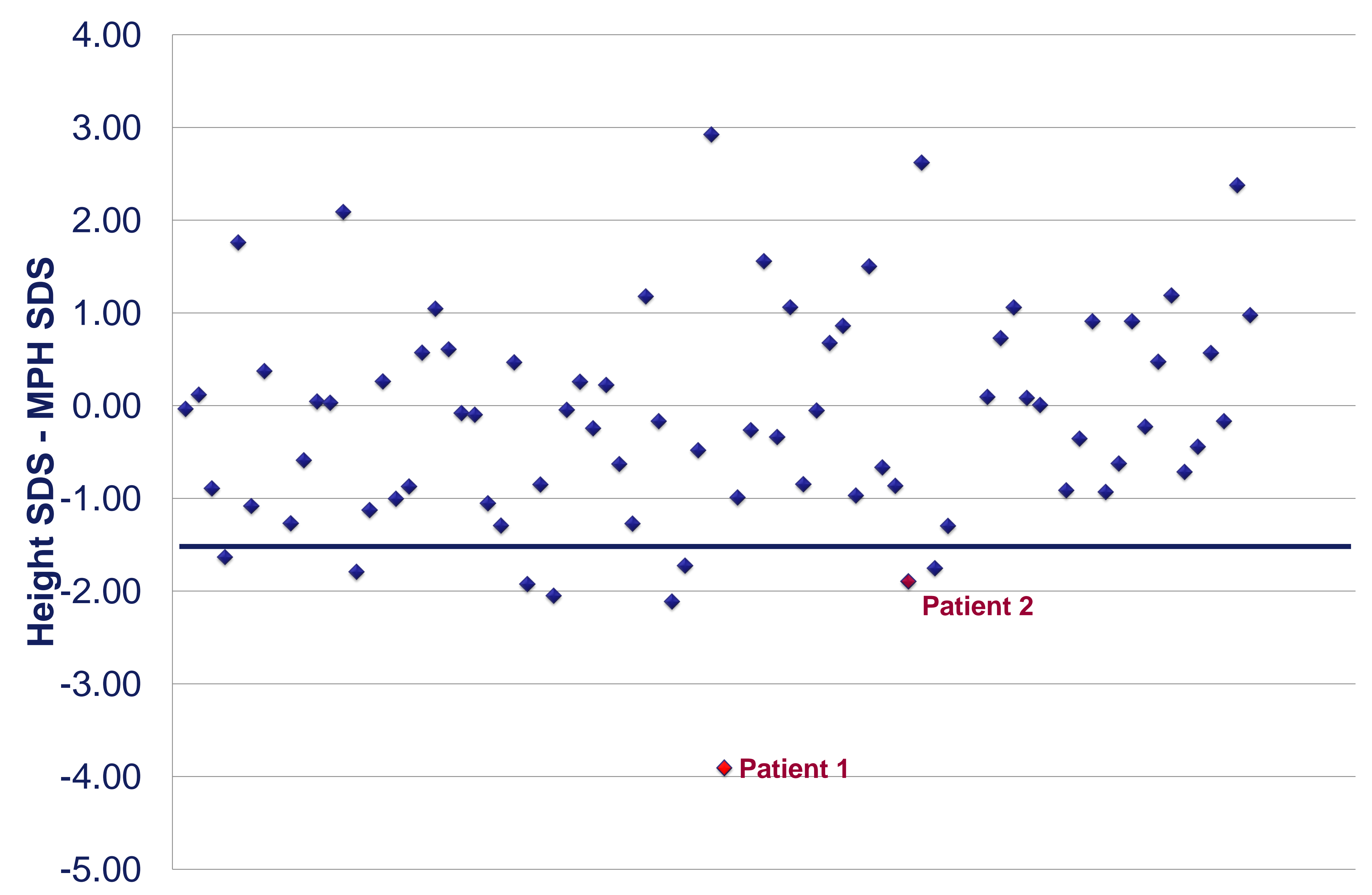

An additional $7 / 83(8.4 \%)$ had height $>1.5 S D$ lower than MPHSDS.

For these 7 patients:

- Median age was $5.14(2.38,7.36)$

- Median height SDS $-0.74(-1.27,-0.28)$

- Median BMI SDS -0.33 (-1.53, 1.68) 